\title{
Time-series analysis of monthly age-specific numbers of newly registered cases of active tuberculosis in Japan from 1998 to 2013
}

\author{
Y. KOHEI ${ }^{1,2}$, A. SUMI ${ }^{1 *}$ AND N. KOBAYASHI ${ }^{1}$ \\ ${ }^{1}$ Department of Hygiene, Sapporo Medical University, Sapporo, Hokkaido, Japan \\ ${ }^{2}$ Department of Nursing, Faculty of Human Science, Hokkaido Bunkyo University, Eniwa, Hokkaido, Japan
}

Received 10 September 2015; Final revision 2 February 2016; Accepted 28 February 2016; first published online 16 March 2016

\section{SUMMARY}

We investigated the seasonality of age-specific tuberculosis (TB) in Japan. To allow the development of TB control strategies for different age groups we used a time-series analysis, including a spectral analysis and least squares method, to analyse the monthly age-specific numbers of newly registered cases of all forms of active TB in Japan from January 1998 to December 2013. The time-series data are reported in 10-year age groups: $0-9,10-19, \ldots, 70-79$, and $\geqslant 80$ years. We defined the contribution ratio of the 1-year cycle, $Q_{1}$, as the contribution of the amplitude of a 1-year cycle to the whole amplitude of the time-series data. The $Q_{1}$ values in the age groups corresponding to adolescence and middle life (10-39 years) and old age ( $\geqslant 70$ years) were high. The peaks in the active TB epidemics for the $\geqslant 70$ years age group occurred in August and September, 1-2 months behind the peaks for the 10-39 years age group (June and July). An active TB epidemic might be attributable to travel by public transport and irregular employment in the 10-39 years age group and immune system suppression by low winter temperatures in the $\geqslant 70$ years age group.

Key words: Age distribution, seasonality, spectral analysis, time-series analysis, tuberculosis.

\section{INTRODUCTION}

Tuberculosis (TB) remains a major public health problem, predominantly affecting Asia and Africa. There were about 9.6 million new cases of TB and 1.5 million deaths from TB in 2014 [1]. To proceed towards the final elimination of TB (defined as $<1$ case of TB per million population), the World Health Organization (WHO) has developed the 'End TB Strategy' [2], with a perspective beyond 2015 [1], to end the global TB epidemic. The targets for 2035 are a $95 \%$ reduction in TB deaths and a $90 \%$ reduction in the incidence of $\mathrm{TB}$, both relative to 2015 levels.

\footnotetext{
* Author for correspondence: Dr A. Sumi, Department of Hygiene, Sapporo Medical University School of Medicine, S-1, W-17, Chuo-ku, Sapporo 060-8556, Japan.

(Email: sumi@sapmed.ac.jp)
}

Understanding the seasonality of TB epidemics may identify potentially modifiable risk factors and suggest new therapeutic measures, and many studies have examined the seasonality of TB epidemics [38]. Previous studies have found that these epidemics occur most frequently in spring in some locations. One major hypothesis explaining the spring peak in TB epidemics is that the likelihood of TB transmission increases in winter, with indoor crowding and poor ventilation, causing the development of primary TB in socially vulnerable people in spring and summer [9]. Another hypothesis proposes that vitamin D deficiency in winter depresses the immune system and increases the risk of TB reactivation in spring or summer [8-10]. A third hypothesis is based on recent evidence that the competence of the immune system varies throughout the year, with significant periodicity 
in immune cell function and the numbers of some subsets of peripheral blood leukocytes [11].

The seasonality of TB epidemics may vary in different situations, such as with age group, sex, geographical area, weather conditions, or economic status [12], and in recent years, the interest in TB in different age groups has increased markedly [13]. In 2012, the WHO included an estimate of childhood TB in their annual report for the first time because children provide the reservoir from which future cases will develop [14]. Without the successful detection and treatment of TB infections and disease in children, any elimination strategy by the WHO will be ineffective [15]. A better understanding of the diversity of TB disease in different age groups is also essential for rational vaccine design, a tool required to reach the WHO targets for 2035 [16].

Researchers have reported that the risk of exposure to TB infection varies with age [17]. A study conducted in Lima, Peru, found that individuals aged 15-44 years were at greatest risk of pulmonary TB [18]. In The Netherlands, TB infections are preferentially transmitted in people of similar ages [19]. More recently, it has been reported that as the transmission and incidence of TB has declined, with more cases arising from older infections than from recent infections, the burden of disease has shifted from younger to older people in India, China, and Japan [17]. Therefore, there is considerable interest in comparing the seasonality of TB in different age groups, to allow the prevention and prediction of $\mathrm{TB}$ epidemics. However, the seasonality of TB epidemics in different age groups has not yet been investigated in detail with the method of time-series analysis.

In Japan, TB surveillance, according to the WHO definition of TB cases, commenced in 1998 [4, 20]. Since then, TB surveillance data on age-specific cases of TB have been collected with a nationwide internet-based infectious diseases reporting system [21]. The incidence of newly registered cases in Japan was $16 \cdot 1 / 100000$ inhabitants in 2013 , so the number of newly registered TB patients exceeded 20 000, defining Japan as having a medium TB burden [22]. By comparison, the rates in many other advanced countries (/100 000) in 2013 were $\leqslant 10$, indicating low TB burden: $3 \cdot 3$ in the United States, 5.0 in Canada, 5.8 in Germany, 6.5 in Switzerland, and 7.0 in Denmark [22]. An investigation of the seasonality of the age-specific TB data collected in Japan could facilitate the prediction of epidemics in each age group and allow Japan to become a low-TB-burden country.
The aim of this study was to investigate the periodic structures in the age-specific cases of active TB in Japan during the period 1998-2013, using a timeseries analysis consisting of a maximum entropy method (MEM) spectral analysis and the least squares method (LSM) [23, 24].

\section{METHODS}

\section{Age-specific active TB case data}

Modes of detecting active TB in patients in Japan are listed in Table 1 . The majority of active TB patients are identified in periodic medical examinations and clinics or hospitals. The periodic medical examinations include a chest X-ray, and consist of individual examinations and mass screenings. Mass screenings are conducted for population groups specified by the TB prevention law: (a) school children and students; (b) inhabitants aged $\geqslant 65$ years; $(c)$ employees in companies, government and municipal offices, schools, hospitals, clinics, birth centres, and social welfare institutions; and $(d)$ inmates of social welfare institutions. If the clinical suspicion of TB persists for 2 weeks in individuals diagnosed at clinics or hospitals, a chest X-ray is performed, together with sputum culture if necessary.

When a physician diagnoses a patient with active TB in any form at a periodic medical examination, clinic, or hospital, the patient is required to notify the public health centre on the same day, according to the TB prevention law [25]. All cases reported to public health centres are regarded as newly notified cases of TB, and may include not only previously untreated cases but also relapsed cases [26].

For cases of active TB newly registered at a public health centre, smear and culture tests are conducted to investigate the bacillus-positive rate. Smear tests are conducted in over $98 \%$ of patients with active TB. The use of culture testing has increased continuously from $48 \%$ to $88 \%$ of cases in the period $1998-2013$ [27].

The numbers of cases of all forms of active TB newly registered each month are collected by the public health centres of all 47 prefectures of Japan and sent to the Japanese Ministry of Health, Labour and Welfare by the local governments. The data are available from Statistics TB [27] and at the website of the National Institute of Infectious Diseases (http:// www.jata.or.jp/rit/ekigaku/) [28].

In this study, a time-series analysis was conducted of the monthly time-series data on the age-specific 
Table 1. Modes of detection of active TB cases in the study population, Japan, 1998-2013

\begin{tabular}{lrr}
\hline \hline & No. & $\%$ \\
\hline $\begin{array}{l}\text { I. Periodic medical examination } \\
\text { 1. Individual examination }\end{array}$ & 82998 & $18 \cdot 0$ \\
2. Mass screening & & \\
$\quad$ (a) School & & \\
$\quad$ (b) Inhabitants & & \\
$\quad$ (c) Employees & & \\
(d) Institution & & \\
II. Clinic/hospital examination & 376900 & $73 \cdot 0$ \\
III. Others/unknown & 10692 & $9 \cdot 0$ \\
\hline \hline
\end{tabular}

numbers of newly registered cases of all forms of active TB in Japan between January 1998 and December 2013. The time-series data are reported in 5 -year age groups. Therefore, we calculated the data for 10-year age groups by summing the data for the 5-year age groups, i.e.: $0-4+5-9,10-14+15-19, \ldots$, $\geqslant 80$ years.

\section{Time-series analysis}

We used a time-series analysis consisting of MEM spectral analysis in the frequency domain and LSM in the time domain. MEM is considered to have a high degree of resolution of spectral estimates. Therefore, MEM spectral analysis allows us to determine short data sequences extremely precisely, such as the infectious disease surveillance data used in this study [29, 30]. The validity of the results obtained with the MEM spectral analysis was confirmed by calculating the optimum least squares fitting (LSF) curve for the time series with LSM.

\section{Spectral analysis}

We assumed that the time-series data $x(t)$ (where $t=$ time) were composed of systematic and fluctuating parts [31]:

$x(t)=$ systematic part + fluctuating part.

The systematic part in equation (1) is regarded as the underlying variation in the original time series, and the fluctuating part, including the undeterministic components such as noise, was obtained as the residual time series when the underlying part was subtracted from the original time series.

To investigate the periodic structures of $x(t)$ in the time-series data for cases of active TB, we performed a spectral analysis based on MEM and calculated the power spectral density (PSD). The formulation of MEM-PSD is described in the appendix of [29].

\section{$L S M$}

The validity of the results of the MEM spectral analysis was confirmed by calculating the LSF curve $X$ $(t)$ for the original time-series data $x(t)$ [equation (1)] with the MEM-estimated periods. The formulation of $X(t)$ is described as:

$X(t)=A_{0}+\sum_{n=1}^{N_{p}} A_{n} \cos \left\{2 \pi f_{n}\left(t+\theta_{n}\right)\right\}$,

which was calculated using LSM for $x(t)$ with unknown parameters $f_{n}, A_{0}$, and $A_{n}(n=1,2,3, \ldots$, $\left.N_{p}\right)$, where $f_{n}\left(=1 / T_{n} ; T_{n}\right.$ is the period $)$ is the frequency of the $n$th component, $A_{0}$ is a constant representing the average value of the time-series data, $A_{n}$ and $\theta_{n}$ are the amplitude and phase of the $n$th component, respectively, and $N_{p}$ is the total number of components.

The optimum values for parameters $A_{0}, A_{n}$, and $\theta_{n}$, $\left(n=1,2, \ldots, N_{p}\right.$ ) in equation (2), but not for $N_{p}$, were exactly determined from the optimum LSF curve calculated using equation (2) with the MEM-estimated period $\left(T_{n}\right)$. The MEM spectral analysis and LSM were performed in MemCalc (Suwa-Trust, Japan) [23]. The detailed theoretical background is described by Ohtomo et al. [24].

The level of reproducibility of $x(t)$ with the optimum LSF curve was evaluated with Pearson's correlation $(\rho)$, using SPSS version $17 \cdot 0 \mathrm{~J}$ software (SPSS Inc., USA).

\section{Contribution ratio}

Based on the results of the MEM spectral analysis, we assigned periodic modes $f_{n}$ on the LSF curve $X(t)$ [equation (2)] that construct the seasonal variations in the original data $x(t)$ [equation (1)]. First, the power of each periodic mode was evaluated with the square of the amplitude, $A_{n}^{2}\left(n=1,2,3, \ldots, N_{p}\right)$ of the $n$th mode constituting the LSF curve $X(t)$. Second, we estimated $R$ corresponding to the power of the residual time series, which was obtained by subtracting the LSF curve from the original data $x(t)$. As a result, the total power of the original time series $Q$ was calculated as:

$Q=\sum_{n=1}^{N_{p}} A_{n}^{2}+R$ 
When both sides of equation (3) are divided by $Q$, we obtain the following normalized relation:

$\sum_{n=1}^{N_{p}} A_{n}^{2} / Q+R / Q=1$,

Where $\sum_{n=1}^{N_{p}}\left(A^{2} n / Q\right)$ and $R / Q$ correspond to the contributions of $\sum_{n=1}^{N_{p}} A^{2} n$ and $R$ to $Q$, respectively. We define the first term on the left-hand side of equation (4) as the 'contribution ratio' [29, 32, 33], which means the contribution $\sum_{n=1}^{N_{p}} A^{2} n$ normalized by $Q$. If $\sum_{n=1}^{N_{p}}\left(A^{2} n / Q\right)$ in the first term becomes large, then the second term, $R / Q$, becomes small.

\section{RESULTS}

\section{Demographic characteristics of active TB cases}

There were 470590 newly registered cases of active TB in Japan between January 1998 and December 2013. The characteristics of those cases are given in Tables 1 and 2 [27]. The proportions of total patients with active TB who were identified during a periodic health examination for TB or at clinics and hospitals were $18 \%$ or $73 \%$, respectively (Table 1 ). Therefore, the overwhelming majority of TB patients were identified at clinics or hospitals. There were more males (64\%) than females $(36 \%)$. The $\geqslant 70$ years age group contributed $44 \cdot 5 \%$ of cases (Table 2 ).

The majority $(80 \%)$ of newly registered cases had pulmonary TB and the remainder had extrapulmonary TB (Table 2). Seventy percent of pulmonary TB cases [corresponding to sputum smear-positive cases $(46 \%)$ plus other bacillus-positive cases $(24 \%)]$ were discharging tubercular bacilli, and sputum smearpositive cases are therefore especially important as sources of infection. Of the sputum smear-positive cases, $4 \%$ had recurrent (re-treated) disease.

\section{Time-series data for age-specific cases of active TB}

Figure $1 a-i$ shows the monthly numbers of newly registered cases of active TB in each age group in Japan between January 1998 and December 2013. The patterns in all the time-series data, except for the $\geqslant 80$ years age group (Fig. $1 a-h$ ), show decreasing trends. However, an increasing trend in the data was observed in the $\geqslant 80$ years age group (Fig. 1i).

For all the age groups shown in Figure 1, relatively large peaks were observed in 1999. These peaks relate to the TB epidemic in that occurred in 1997, when the number of new cases increased for the first time in 38
Table 2. Characteristics of active TB cases in the study population, Japan, 1998-2013

\begin{tabular}{lrr}
\hline \hline & No. & $\%$ \\
\hline All active TB cases & 470590 & 100 \\
Sex & & \\
$\quad$ Male & 301143 & $64 \cdot 0$ \\
$\quad$ Female & 169447 & $36 \cdot 0$ \\
Age, years & & \\
$\quad<14$ & 2132 & $0 \cdot 5$ \\
15-39 & 82219 & $17 \cdot 5$ \\
40-69 & 176552 & $37 \cdot 5$ \\
TB diagnosis & 209687 & $44 \cdot 5$ \\
Pulmonary TB & & \\
$\quad$ Male & 377116 & $80 \cdot 1$ \\
$\quad$ Female & 250301 & $66 \cdot 0$ \\
$\quad$ Classification & 126815 & $34 \cdot 0$ \\
$\quad$ Sputum smear positive & 174525 & $46 \cdot 0$ \\
$\quad$ New & 159042 & $42 \cdot 0$ \\
$\quad$ Retreatment & 15483 & $4 \cdot 0$ \\
$\quad$ Other bacillus positive & 90418 & $24 \cdot 0$ \\
$\quad$ Bacillus negative/unknown & 112173 & $30 \cdot 0$ \\
Extrapulmonary TB & 93474 & $19 \cdot 9$ \\
Male & 50842 & $54 \cdot 0$ \\
Female & 42632 & $46 \cdot 0$ \\
\hline \hline & & \\
\hline
\end{tabular}

years and the prevalence rate increased for the first time in 43 years. In July 1999, the Japanese Government declared a state of emergency concerning the TB epidemic of 1997 to raise public awareness of the increase in TB in the country [25]. In the month following this declaration, the number of registered TB cases increased sharply, resulting in relatively large peaks in 1999 for all age groups, as shown in Figure 1.

\section{Setting up the time-series data for age-specific cases of active TB}

The PSDs, $P(f)$ s ( $f[1 /$ year $]$ : frequency), were calculated for all the time-series data shown in Figure 1, and the results are shown in Figure $2(f \leqslant 1 \cdot 5)$. In each PSD (Fig. 2), the longest period appears as a prominent peak at a frequency position longer than the length of the original data (16 years, from January 1998 to December 2013), i.e. a $27 \cdot 6$-year period for the $0-9$ years age group, for example (Fig. 2a). Using this 27.6-year period, the long-term trend in the data for the $0-9$ years age group was estimated by calculating the LSF with equation (2). The results are shown in Figure $1 a$. The long-term trends for the other age groups were estimated with the same procedure. As 
(a)

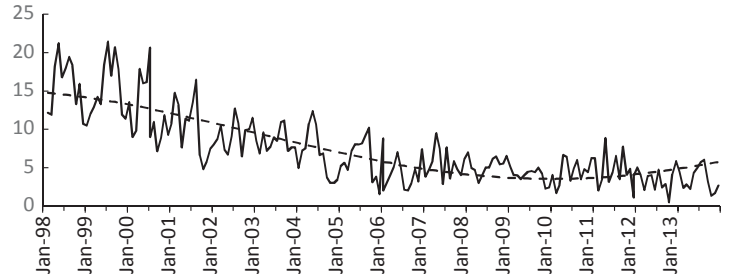

(f) 80

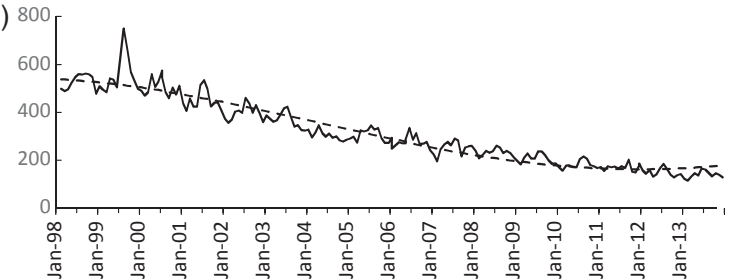

(b) 1

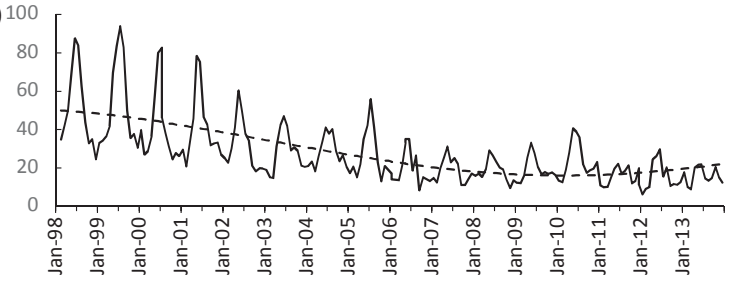

(g) 90

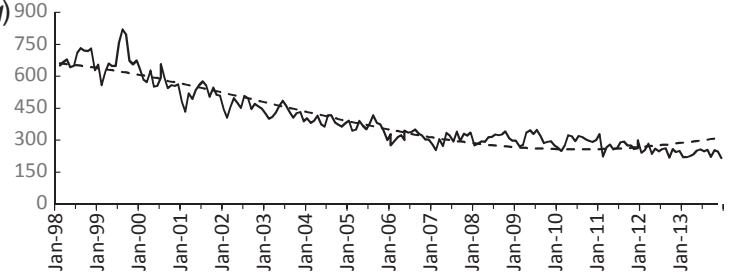

(c) 50

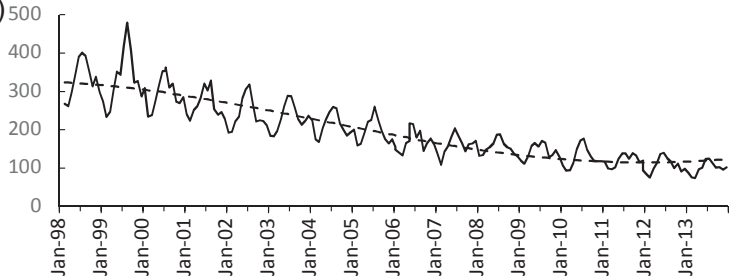

(h) 100

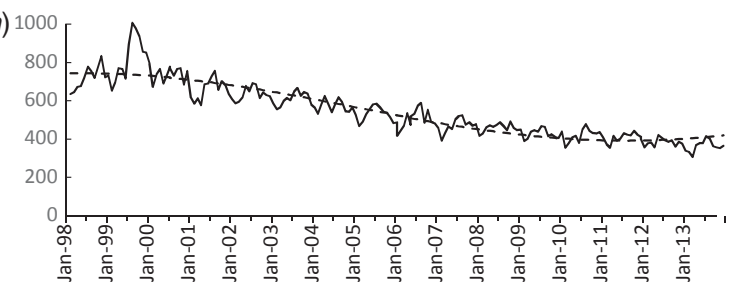

(d)
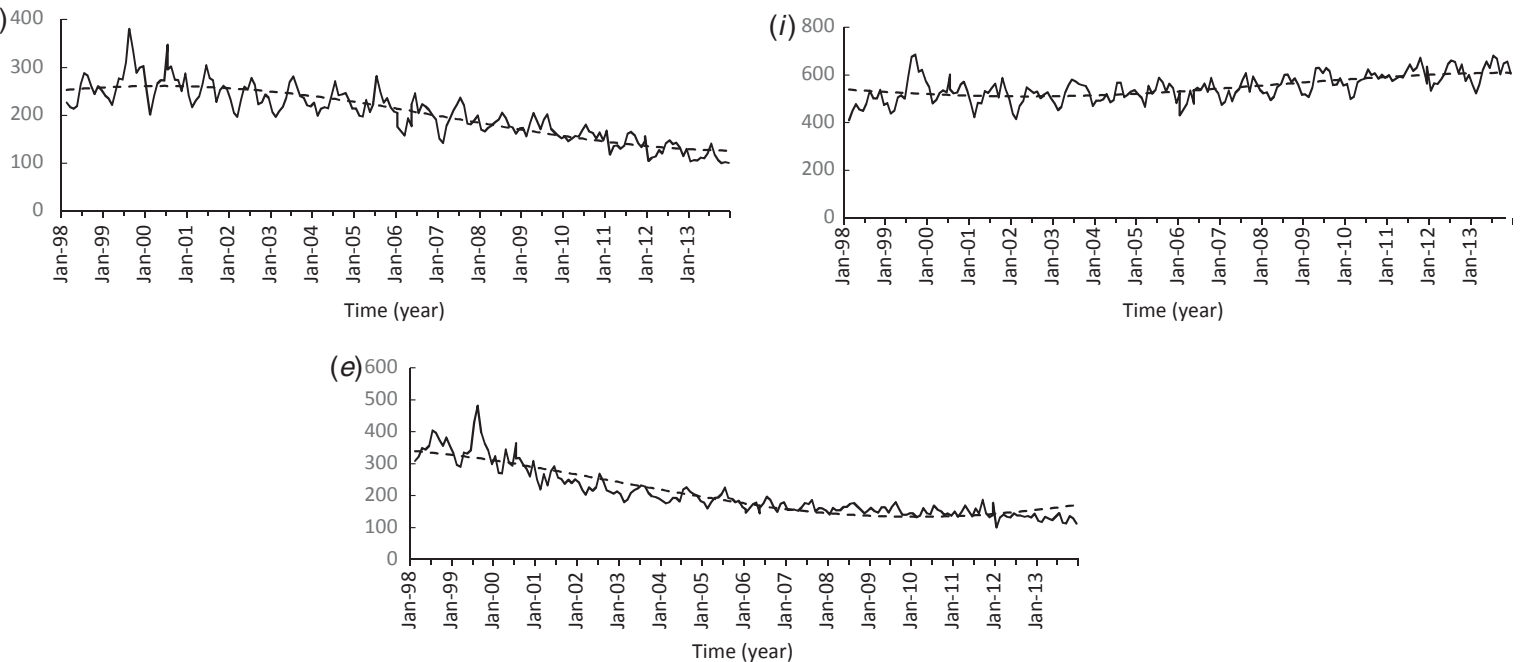

Fig. 1. Monthly age-specific numbers of newly registered cases of all forms of active TB in Japan between January 1998 and December 2013 (solid line), and the long-term trends (dashed line) in different age groups: $(a)$ 0-9, (b) 10-19, (c) 2029, (d) 30-39, (e) 40-49, (f) 50-59, $(g) 60-69,(h) 70-79$, and $(i) \geqslant 80$ years.

shown in Figure 1, the LSF curves for all the age groups reproduced the long-term trends in the data well. The LSF curves were removed from the original data to obtain the residual data (Fig. 3a-i). The seasonality of the age-specific active TB data was investigated using the residual data.

\section{Spectral analysis}

The PSDs were calculated for the residual data (Fig. 3), and the semi-log-scale plots $(f \leqslant 1 \cdot 5)$ are shown in Figure 4. In all the PSDs, prominent spectral peaks occur at $f=1 \cdot 0\left(=f_{1}\right)$, corresponding to a 1-year period, i.e. the seasonal cycle of disease epidemics.

\section{Contribution ratio of the 1-year periodic mode}

Figure 5 shows plots of the value of $Q_{1}$ against the data for the age groups of patients with active TB in Japan in 1998-2013, with the 95\% confidence intervals (CIs). The curve for $Q_{1}$ increases when $0 \leqslant$ age $\leqslant 39$ and reaches $Q_{1}=0.87$ (95\% CI $0.81-0.93$ ). 

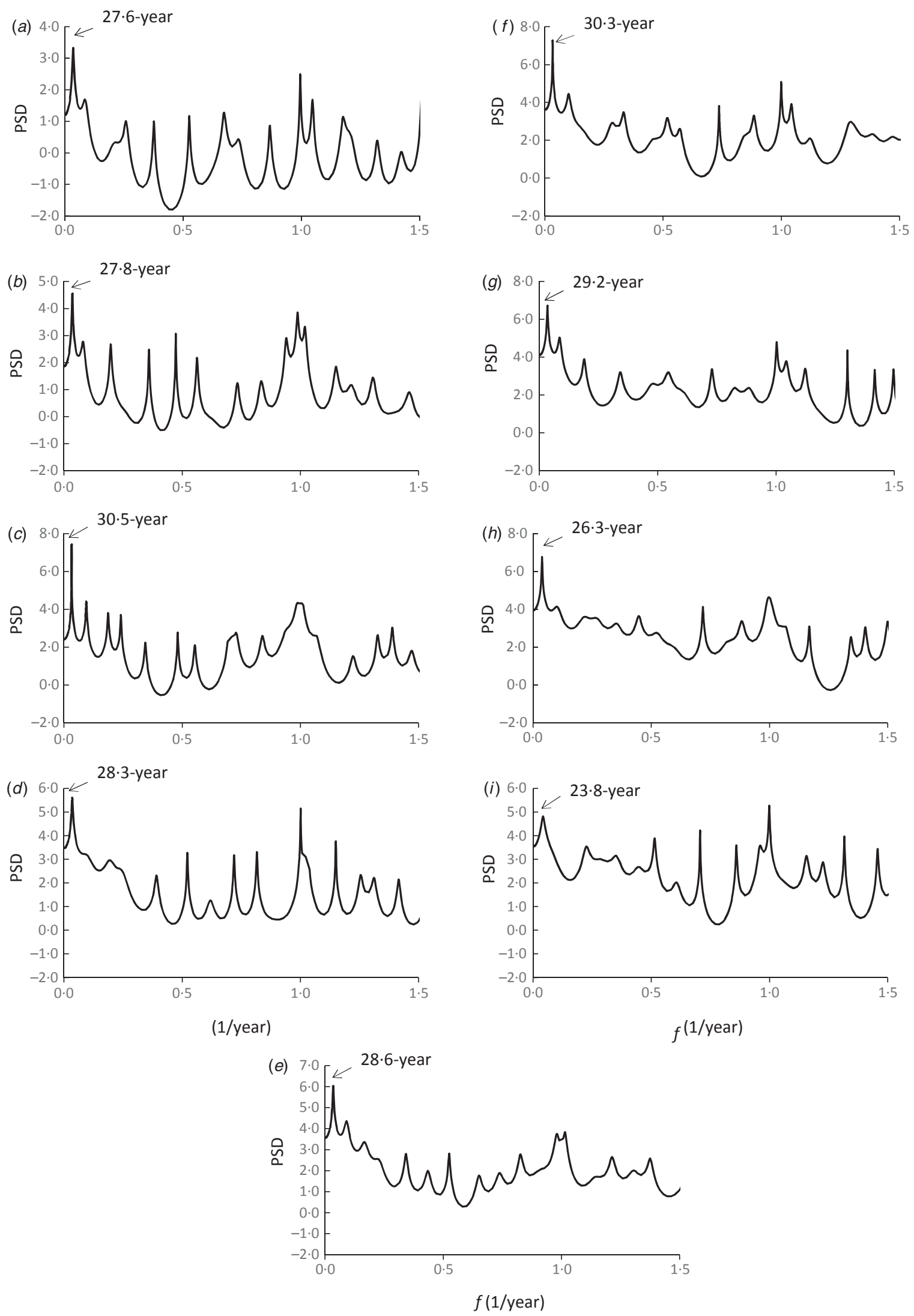

Fig. 2. Power spectral densities (PSDs) calculated from the active TB data $(f \leqslant 1 \cdot 5)$ for different age groups: $(a) 0-9$, $(b)$ 10-19, (c) 20-29, (d) 30-39, (e) 40-49, (f) 50-59, (g) 60-69, (h) 70-79, and (i) $\geqslant 80$ years. 

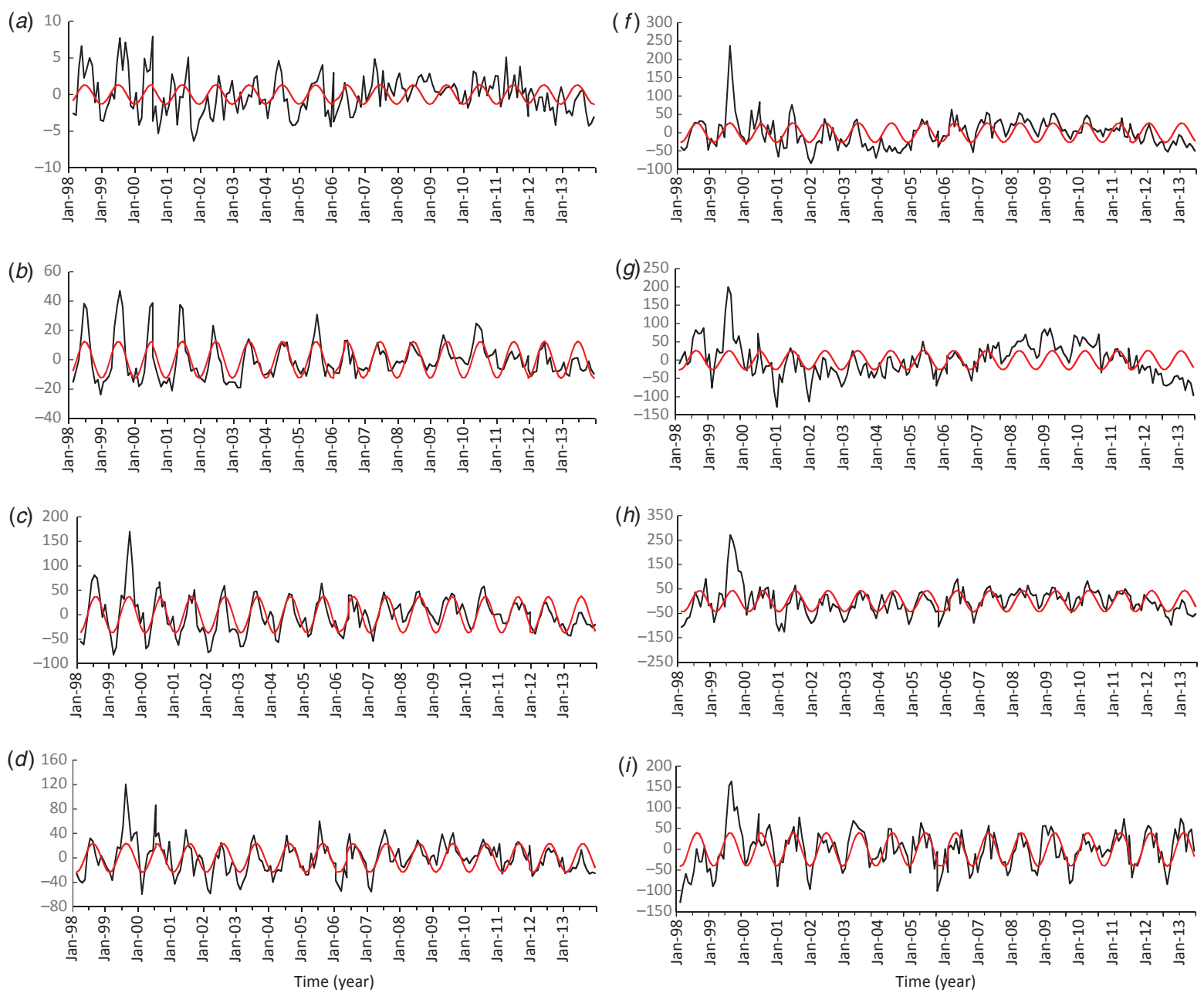

(e)

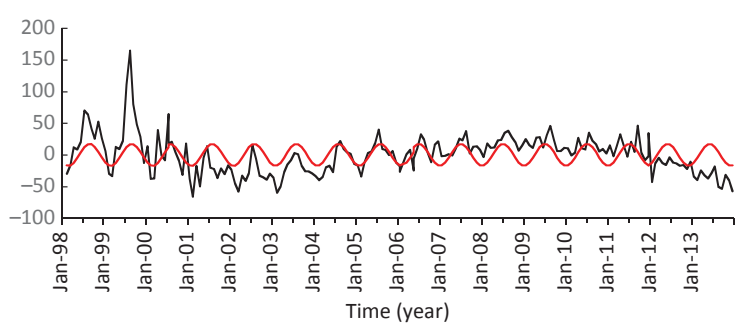

Fig. 3. Residual time-series data obtained by subtracting the long-term trends in the active TB data from the active TB data (solid line), and the least squares fitting curves calculated with 1-year cycles (red line) for the different age groups: $(a)$ $0-9$, (b) 10-19, (c) 20-29, (d) 30-39, (e) 40-49, (f) 50-59, (g) 60-69, (h) 70-79, and (i) $\geqslant 80$ years.

Thereafter, the value of $Q_{1}$ decreases to $0 \cdot 28$ when $40 \leqslant$ age $\leqslant 49(95 \%$ CI $0 \cdot 11-0 \cdot 45)$, and remains relatively constant around $0 \cdot 3$ when $40 \leqslant$ age $\leqslant 69$. The $Q_{1}$ value then increases at ages $\geqslant 70$ years and reaches $Q_{1}=0.57(95 \%$ CI $0.49-0.65)$ at ages $\geqslant 80$ years.

\section{Peak months in the time-series data for the 10-39 and $\geqslant 70$ years age groups}

To investigate the peak months of active TB epidemics, we focused on ages $10-39$ years and $\geqslant 70$ years, in which the $Q_{1}$ values were relatively high (Fig. 5). Figure $6(a, b)$ summarize the time-series data for age groups 10-39 years (Fig. 1b-d) and $\geqslant 70$ years (Fig. $1 h-i)$, respectively.

Preparation of an estimation of the peak month of the time-series data

The time-series analysis of the data in Figure $6(a, b)$ was conducted with the same procedure used for the original data for each age group (Fig. 1a-i). First, a 

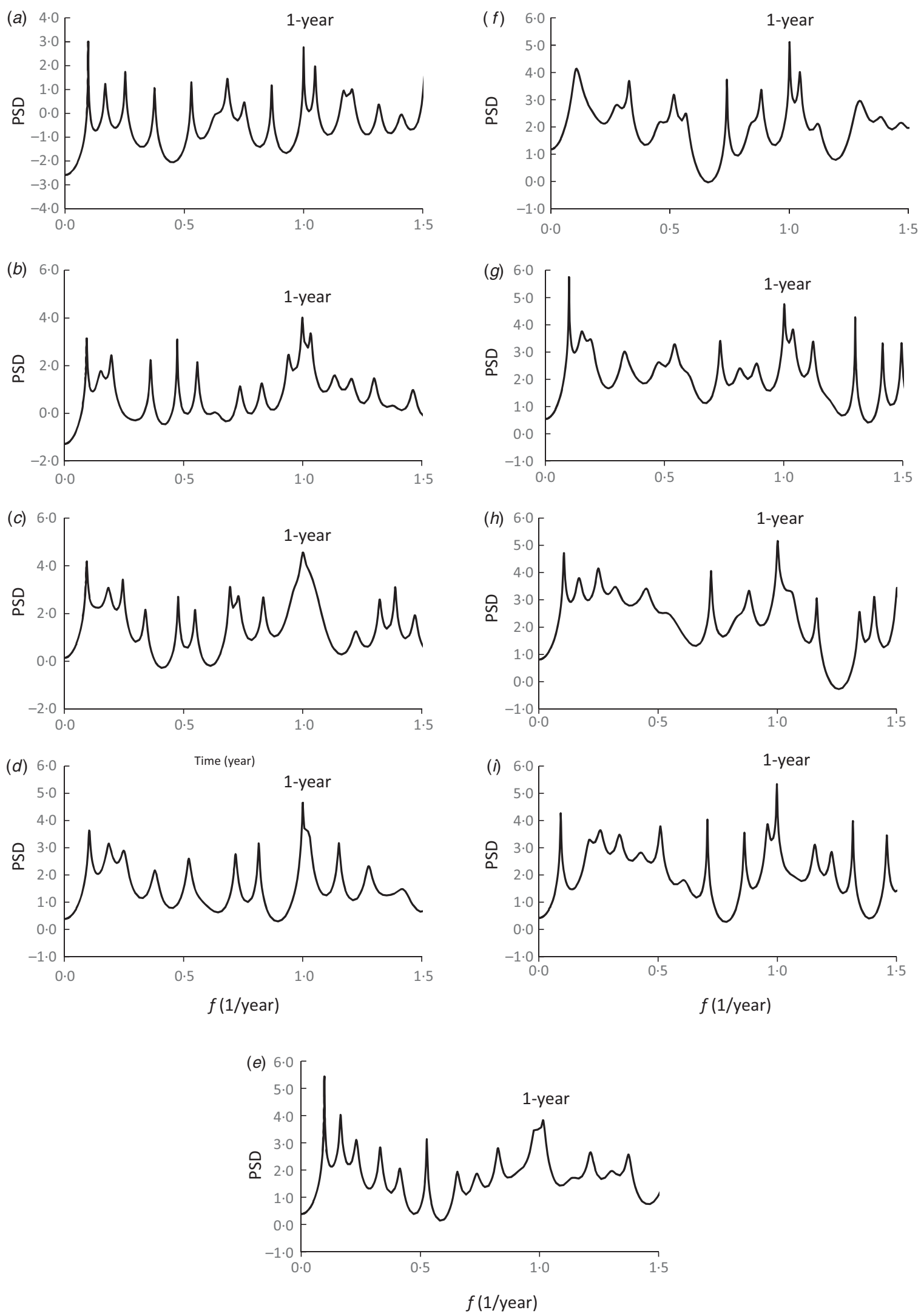

Fig. 4. Power spectral densities (PSDs) obtained from the residual data $(f \leqslant 1 \cdot 5)$ for the different age groups: (a) $0-9$, $(b)$ 10-19, (c) 20-29, (d) 30-39, (e) 40-49, (f) 50-59, (g) 60-69, (h) 70-79, and $(i) \geqslant 80$ years. 


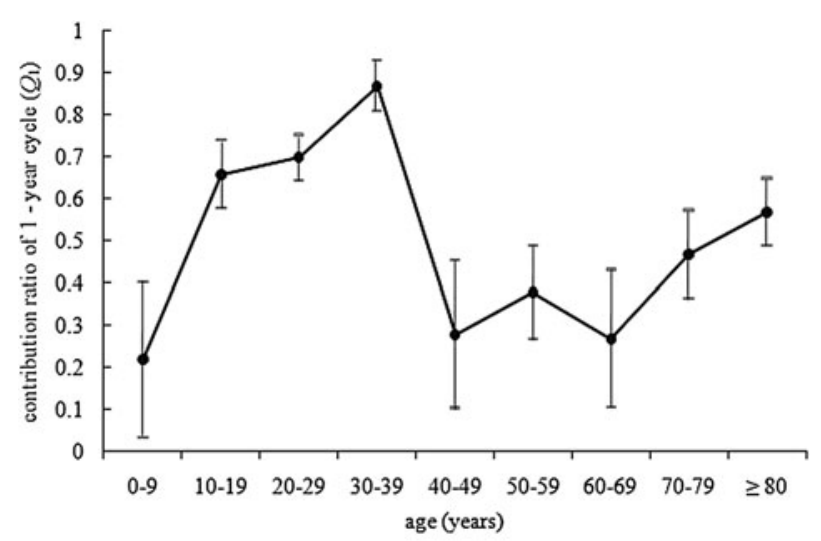

Fig. 5. Contribution ratio of a 1-year cycle $\left(Q_{1}\right)$ against age (years). Bars represent 95\% confidence intervals (CIs). The CIs were obtained from the $Q_{1}$ values for eight segment data (length 2 years) of the residual data in Figure 3.

spectral analysis of the original data was performed (Fig. 6a, $b$ ) and the long-term periods were determined from the PSDs of the original data: $30 \cdot 8$ years and 22.5 years for age groups 10-39 years (Fig. 6c) and $\geqslant 70$ years (Fig. $6 d$ ), respectively. Next, the long-term trends were calculated as the LSF curves with equation (2) (Fig. 6a, b). These trends were removed by subtracting the LSF curves from the original data, and the residual data were obtained (Fig. $6 e, f$ ). The MEM-PSD of the residual data was then calculated (Fig. $6 g, h$ ). In each PSD, 10 spectral peak frequency modes were selected, and these are summarized with the corresponding periods and intensities (powers) of the spectral peaks in Table 3 .

\section{Assignment of fundamental modes of the time-series data}

To obtain the optimum LSF curve for the residual data (Fig. $6 e, f$ ), we assigned the fundamental modes constructing the underlying variation in equation (1). We then calculated the contribution ratio [equation (3)] with the variation in $N_{p}$ by adding 10 MEM-estimated periods (Table 3) to the LSF curve [equation (2)], one by one in order of the magnitude of the power of the spectral peak frequency. The results are shown in Figure 6(i,j) for age groups 1039 and $\geqslant 70$ years, respectively. Both figures show inflection points at $N_{p}=3$. We then separated the contributions of the 10 periods into two parts at $N_{p}=3$ : the underlying variation and the fluctuating part described in equation (1). For the residual data for age groups $10-39$ years (Fig. 6e) and $\geqslant 70$ years
(Fig. 6f), we assigned the three periods as the fundamental modes: $10 \cdot 1,1 \cdot 0$, and $0 \cdot 5$ years for the $10-39$ years age group and $9 \cdot 2,3.9$, and 1.0 years for the $\geqslant 70$ years age group.

\section{Determination of the peak months from the time-series data}

Using the three fundamental modes, we calculated the LSF curve with equation (2) for each age group (Fig. $6 e, f$ ). The values for $\rho$ between the residual data and the LSF curves were 0.83 and 0.74 in Figure $6(e, f)$, respectively. The peaks in the LSF curve for the 10-39 years age group (Fig. 6e) were observed in June and July. For the $\geqslant 70$ years age group (Fig. 6f), the peaks in the LSF curve were observed in August and September.

\section{DISCUSSION}

In this study, the contribution ratio of the 1-year cycle $\left(Q_{1}\right)$ of active TB cases in Japan varied according to age group (Fig. 5). The $Q_{1}$ values for the adolescent and middle-aged group (10-39 years) and the elderly group ( $\geqslant 70$ years) were relatively high (Fig. 5). The following explanations are proposed based on the environmental and biological conditions in Japan: (i) an increase in the length of time performing activities in poorly ventilated and humid environments; (ii) vitamin D deficiency resulting from limited exposure to sunlight; and (iii) seasonal immunocompetence.

The $Q_{1}$ value calculated for the 10-39 years age group (Fig. 5) can be interpreted in terms of '(i) an increase in the length of time performing activities in poorly ventilated and humid environments'. In 2010, the proportion of TB patients aged 20-40 years was higher in urban areas than in rural areas, although $\geqslant 65 \%$ of TB patients in Japan were elderly [27]. In Tokyo, 1077 (35.4\%) of the 3075 newly registered patients were in the 20-40 years age group in 2010, and the prevalence of TB (/100 000 population) was 19 , which was the highest prevalence in this age group in any prefecture in Japan [27]. It has been suggested that foreigners from countries with a high TB burden or from socially and economically vulnerable countries account for many patients in the 20-40 years age group in Japan [27]. However, the 2009 survey of TB in Japan found that in the newly registered TB patients aged 20-49 years, foreign nationals accounted for only $12 \cdot 1 \%$, and more than half the patients were Japanese workers, students, or 
(a)
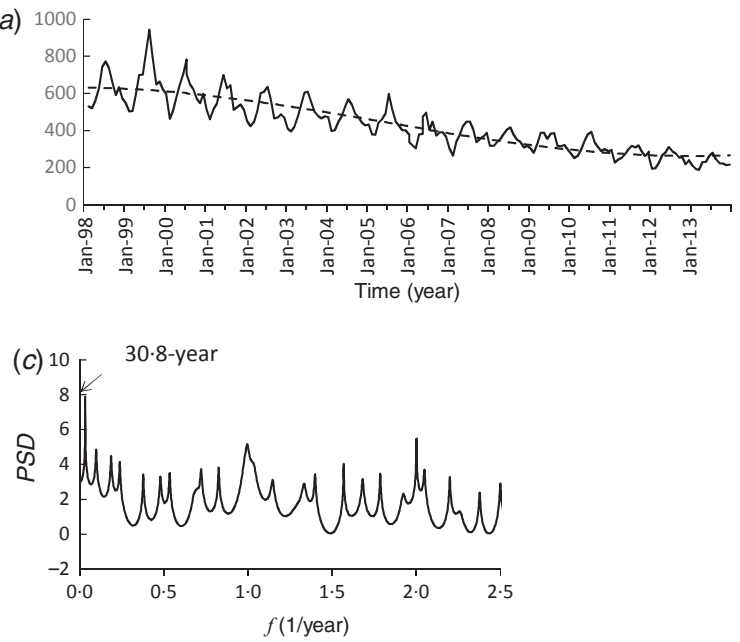

(e)
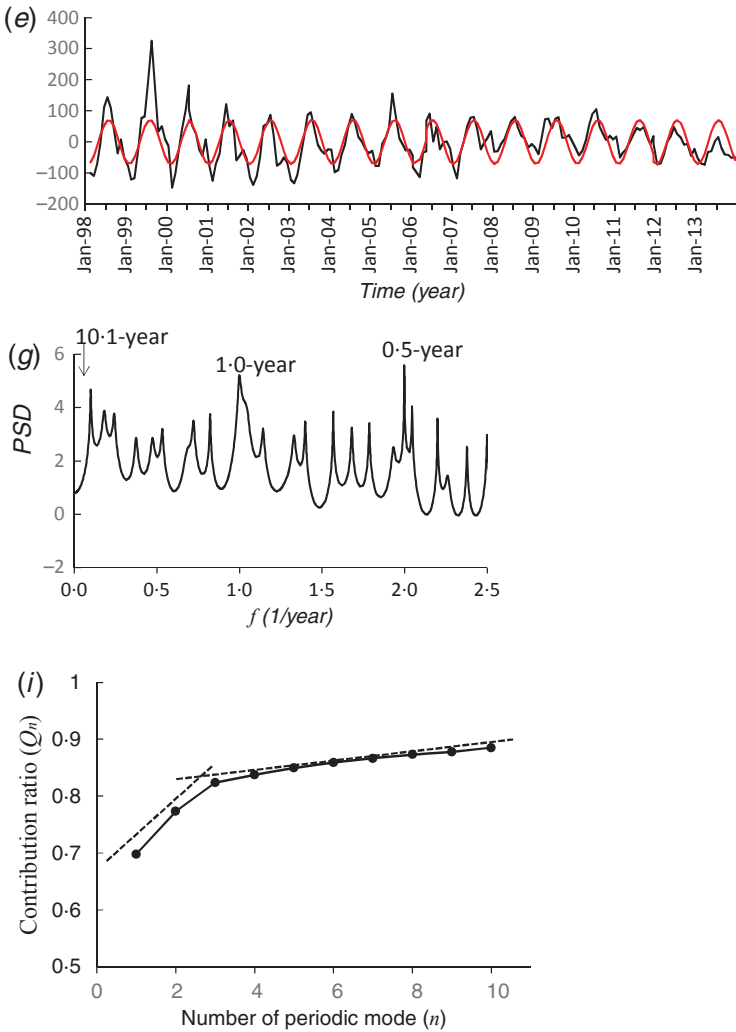
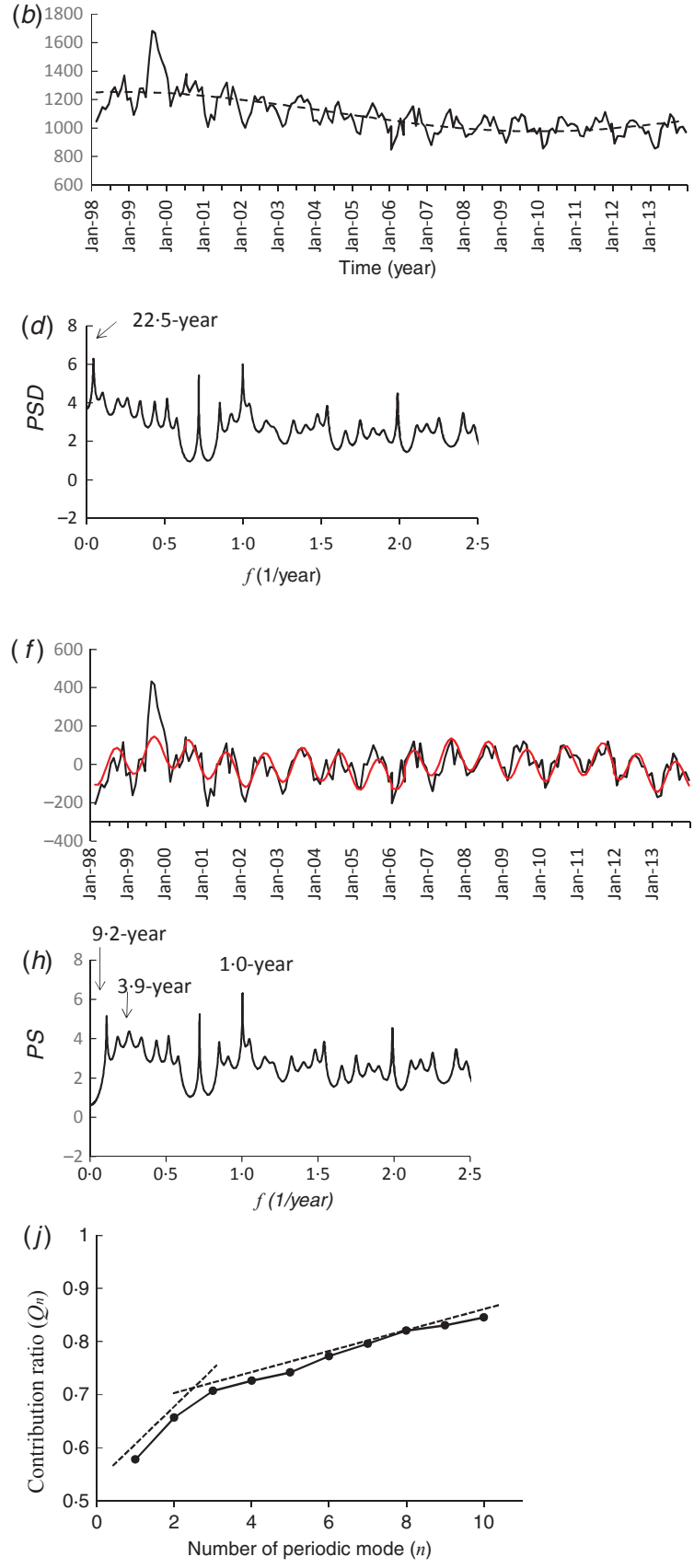

Fig. 6. Seasonality of the $10-39$ and $\geqslant 70$ years age groups. $(a, b)$ Active TB data (solid line) and long-term trends (dashed line) for the 10-39 and $\geqslant 70$ years age groups, respectively; $(c, d)$ power spectral densities (PSDs) for the active TB data for the 10-39 and $\geqslant 70$ years age groups, respectively; $(e, f)$ residual time-series data obtained by subtracting the long-term trends in the active TB data from the active TB data (solid line), and the least squares fitting curves calculated with three periodic modes (red line); $(g, h)$ PSDs for the residual data for the 10-39 and $\geqslant 70$ years age groups, respectively; $(i, j)$ contribution ratios, $Q_{n}$, against numbers of periodic modes $n\left(=1,2, \ldots, N_{p}\right)$ for the $10-39$ and $\geqslant 70$ years age groups, respectively.

homemakers [27]. Hoshino et al. [34] reported that in urban areas of Japan, the risk of infection with TB increases with the use of public transport and that contact surveys of TB outbreaks related to public transport are required. A study conducted in Lima,
Peru, also indicated that public transport was an important risk factor for TB [18]. That study also reported that urban living involves small, poorly ventilated, and overcrowded accommodation, which is a risk factor for TB. In Japan, because the recent 
Table 3. Characteristics of the ten dominant spectral peaks shown in Figure $6(g, h)$

\begin{tabular}{|c|c|c|c|c|c|}
\hline \multicolumn{3}{|c|}{$10-39$ years age group } & \multicolumn{3}{|c|}{$\geqslant 70$ years age group } \\
\hline$F$ & Period (year) & Power & $f$ & Period (year) & Power \\
\hline $0 \cdot 10$ & $10 \cdot 11^{*}$ & $21 \cdot 03$ & $0 \cdot 11$ & $9 \cdot 20^{*}$ & $30 \cdot 62$ \\
\hline $0 \cdot 18$ & $5 \cdot 52$ & $15 \cdot 74$ & $0 \cdot 18$ & $5 \cdot 47$ & $23 \cdot 20$ \\
\hline $0 \cdot 24$ & $4 \cdot 12$ & $12 \cdot 98$ & $0 \cdot 26$ & $3 \cdot 88^{*}$ & $27 \cdot 05$ \\
\hline 0.72 & $1 \cdot 39$ & $8 \cdot 47$ & $0 \cdot 34$ & $2 \cdot 96$ & $20 \cdot 78$ \\
\hline $0 \cdot 82$ & $1 \cdot 22$ & $11 \cdot 03$ & $0 \cdot 44$ & $2 \cdot 29$ & $16 \cdot 94$ \\
\hline $1 \cdot 00$ & $1 \cdot 00^{*}$ & $68 \cdot 80$ & $0 \cdot 52$ & 1.93 & $19 \cdot 79$ \\
\hline $2 \cdot 00$ & $0 \cdot 50^{*}$ & $28 \cdot 68$ & $1 \cdot 00$ & $1 \cdot 00^{*}$ & $87 \cdot 27$ \\
\hline $2 \cdot 04$ & $0 \cdot 49$ & $11 \cdot 65$ & $1 \cdot 05$ & $0 \cdot 95$ & 23.95 \\
\hline $2 \cdot 99$ & $0 \cdot 33$ & $11 \cdot 42$ & $2 \cdot 99$ & $0 \cdot 33$ & $25 \cdot 09$ \\
\hline $4 \cdot 20$ & $0 \cdot 24$ & $7 \cdot 89$ & $4 \cdot 18$ & $0 \cdot 24$ & $18 \cdot 82$ \\
\hline
\end{tabular}

* The assigned fundamental nodes.

recession caused a deterioration of the employment situation, many young people are working in irregular employment. Of these, day labourers remain in urban areas to find employment and use saunas, capsule hotels, internet cafes, and so on, where many vagrant people gather to sleep and mass infections of TB occur [27]. To reduce the cases of TB in the age group corresponding to adolescence and middle life, it is imperative that a system for conducting periodic medical examinations be developed, not only for regularly employed workers but also for those irregularly employed, including day labourers.

The $Q_{1}$ value for the $\geqslant 70$ years age group (Fig. 5) can be interpreted in terms of '(iii) seasonal immunocompetence'. To determine the environmental factor that triggers seasonal changes in immune cell numbers in humans, several studies have examined the potential role of temperature as a mediator of seasonal fluctuations in immunity [35]. The $\geqslant 70$ years age group is not only a high-risk population for TB but also highly vulnerable to extreme temperatures [36]. The immune systems of the elderly $(\geqslant 70$ years) might be suppressed by low temperatures in the winter in Japan, resulting in their infection with TB, increasing the numbers of notifications in the spring and summer.

The high numbers of notifications in adolescents and the middle-aged (10-39 years) in spring and summer (June-July; Fig. 6e) demonstrate the seasonality of active TB in this age group. The spring and summer peaks in active TB epidemics can be explained as follows. Cell-mediated immunity develops 2-6 weeks after the primary infection with
Mycobacterium tuberculosis, and active TB develops if the immune system fails to control bacterial multiplication [4]. In Japan, the time interval between the onset of symptoms of active TB and its diagnosis is about 13 weeks [4]. Therefore, theoretically, people who are infected in autumn or winter will develop active TB shortly after their infection is diagnosed, on average 3-4 months after the first onset of symptoms, resulting in higher numbers of notifications in spring and summer. This pattern of TB pathogenesis has also been observed in The Netherlands [37] and Peru [7]. The seasonality of active TB in the elderly $(\geqslant 70$ years) is shown in Figure $6 f$, and the peaks on the LSF curve occur 1-2 months behind the peaks on the curve for the 10-39 years age group. This supports the notion that in the elderly ( $\geqslant 70$ years), the interval between the onset of symptoms of active TB and its diagnosis tends to be longer than in other age groups [27]. This delay in the diagnosis of TB may occur because in many elderly patients, nonrespiratory symptoms, such as fever, loss of appetite, general malaise, and weight loss, are not attributed to TB [27]. This delay in the diagnosis of TB also occurs when there is a shadow on a chest X-ray, because it is often interpreted as a sign of pneumonia or lung cancer rather than as TB [27]. It is important for medical doctors to carefully observe any changes in the delicate condition of elderly patients and to diagnose TB rapidly [27].

A case study indicated that in patients aged $\geqslant 65$ years, $28.5 \%$ died within 1 year of the start of $\mathrm{TB}$ treatment, and this proportion increases with increasing age [27]. This is partly because a large proportion of TB patients have complications, such as malignant tumour, chronic renal failure, or diabetes. These complications make it difficult for elderly patients to live at home, even if they are discharged from hospital. Japan will be unable to ignore its ageing society in the future. In terms of geriatric issues [38], it is necessary for elderly patients to engage long-term care insurance services to prevent the deterioration of their activities of daily living and to provide assistance with medication after hospital discharge.

In this study, an increasing trend in the newly registered case of active TB was observed in the $\geqslant 80$ years age group (Fig. 1i). This increasing trend was caused by an increase in the number of people in this age group from 1998 to 2013. However, the incidence rate (corresponding to the number of newly registered cases/100 000 population) in the $\geqslant 80$ years age group gradually decreased, as it also did in other age groups, 
but was higher $(79 \cdot 5)$ than in any other age group in 2013: $0 \cdot 4$ (0-9 years), 1.6 (10-19 years), 9.1 (20-29 years), $7 \cdot 9$ (30-39 years), 8.3 (40-49 years), 10.8 (5059 years), $15 \cdot 4$ (60-69 years) and 31·4 (70-79 years). The high incidence rate $(79 \cdot 5)$ in the $\geqslant 80$ years age group can be explained as follows. TB epidemics during the first half of the 20th century in Japan and the lower socioeconomic conditions before the World War II [39] were responsible for high infection rates in these years. Therefore, the majority of currently elderly people were infected during their early lives in this period. In most cases of TB in the elderly, the disease develops when the bacterium that was contracted during their youth and has lain dormant within their bodies for decades is reactivated because the immune system is suppressed by diabetes, cancer, treatment with immunosuppressive drugs, or old age [40].

Japan has been conducting the universal bacille Calmette-Guérin (BCG) vaccination of infants since 1951, with the revaccination of children in the first year of primary school and the first year of junior high school since 1954. Japan discontinued the double inoculation with BCG in April 2003 in response to a revision in the law. At present, infants are vaccinated against TB before they are 1 year old, usually between 5 and 8 months of age. The rate of BCG vaccination in Japan in 2013 was 97\% [27]. Consequently, since 2006, only one case of tuberculous meningitis has been reported in infants aged $0-4$ years in Japan [27]. On the other hand, it was reported that, when infants are inoculated with the BCG vaccine at the time of TB infection, the disease develops in a greater number of children and the disease was aggravated in its early stage [27]. Understanding the Koch phenomenon after BCG vaccination, which can include fever, swelling, and a purulent lesion, is extremely important because it provides an opportunity to diagnose infants with TB infection.

In this study, the number of males $(66 \%)$ with pulmonary TB was greater than the number of females (34\%) (Table 2). This differs from the results of a Peruvian study in which there was no sex-related difference in the frequency of pulmonary TB [18]. A possible explanation for this discrepancy could involve the social and behavioural differences between males and females in Japan. Of the male patients with pulmonary $\mathrm{TB}$ in $2013,54 \%$ were aged $\geqslant 70$ years. Men of this generation were more likely to work outside and to acquire TB infections during their youth than were women of the same age. Men of this generation are also more likely than women to smoke and consume alcohol, which can stimulate a latent TB infection to become symptomatic [27].

A limitation of this study was that our results cannot be interpreted in terms of '(ii) vitamin D deficiency resulting from limited exposure to sunlight'. It was recently reported that vitamin D levels depend on the angle of incidence of sunlight, and therefore on latitude [6]. However, if vitamin D does play a role in the seasonality of TB, it may be through a mechanism that is not yet understood [3]. Japan extends from latitude $45^{\circ} \mathrm{N}$ to $23^{\circ} \mathrm{N}$, so the intensity of incident sunlight and vitamin levels vary widely. To determine the effects of vitamin levels on the prevalence of TB in Japan, a systematic study of the effects of latitude on the seasonality of TB throughout Japan will be required. The seasonality of active TB epidemics might also have multiple other causes [12]. An investigation of the correlation between weather conditions and active TB epidemics might be especially important in determining whether the immune systems of elderly people ( $\geqslant 70$ years) are suppressed by low temperatures in winter [35, 36]. Such information would allow us to better control active TB epidemics in the elderly.

In conclusion, we have identified differences in the seasonality of the monthly age-specific numbers of newly registered cases of active TB in Japan (Fig. 5). We have demonstrated that individuals aged 10-39 and $\geqslant 70$ years are particularly important contributors to the seasonality of active TB epidemics, and that the periodic structures of active TB epidemics differ in these two age groups. To control active TB epidemics, it is necessary to investigate the periodic structures in the temporal patterns of active TB in each age group, as in the present study. We anticipate that the present method of time-series analysis, including an MEM spectral analysis and LSM, will have utility in further studies of the seasonality of agespecific TB.

\section{ACKNOWLEDGEMENTS}

This study was supported in part by a Grant-in-Aid for Scientific Research (grant no. 25460769) from the Ministry of Education, Culture, Sports, Science and Technology of Japan.

\section{DECLARATION OF INTEREST}

None. 


\section{REFERENCES}

1. World Health Organization. Global tuberculosis report 2015. Geneva: World Health Organization (http://www. who.int/tb/publications/global_report/en/). Accessed 10 December 2015.

2. World Health Organization. The end TB strategy. Geneva: World Health Organization (http://www.who.int/ tb/End_TB_brochure.pdf?ua=1). Accessed 10 December 2015.

3. Willis MD, et al. Seasonality of tuberculosis in the United States, 1993-2008. Clinical Infectious Diseases 2012; 54: 1553-1560.

4. Nagayama N, Ohmori M. Seasonality in various forms of tuberculosis. International Journal of Tuberculosis and Lung Disease 2006; 10: 1117-1122.

5. Naranbat N, et al. Seasonality of tuberculosis in an Eastern Asian country with an extreme continental climate. European Respiratory Journal 2009; 34: 921-925.

6. MacLachlan JH, Lavender CJ, Cowie BC. Effect of latitude on seasonality of tuberculosis, Australia, 2002-2011. Emerging Infectious Diseases 2012; 18: 1879-1881.

7. Wingfield T, et al. The seasonality of tuberculosis, sunlight, vitamin D, and household crowding. Journal of Infectious Diseases 2014; 210: 774-783.

8. Thorpe LE, et al. Seasonality of tuberculosis in India: is it real and what does it tell us? Lancet 2004; 364: 16131614.

9. Fares A. Seasonality of tuberculosis. Journal of Global infectious Diseases 2011; 3: 46-55.

10. Wacker M, Holick MF. Vitamin D-Effects on skeletal and extra skeletal health and the need for supplementation. Nutrients 2013; 5: 111-148.

11. Nelson RJ, et al. Seasonal Patterns of Stress, Immune Function, and Disease. New York: Cambridge University Press, 2005, pp. 58-88.

12. Sloot R, et al. Risk of tuberculosis after recent exposure: a 10 year follow-up study of contacts in Amsterdam. American Journal of Respiratory and Critical Care Medicine 2014; 190: 1044-1052.

13. Newton SM, et al. Paediatric tuberculosis. Lancet Infectious Diseases 2008; 8: 498-510.

14. World Health Organization. Global tuberculosis report 2012. Geneva: World Health Organization (http://apps. who.int/iris/bitstream/10665/75938/1/9789241564502_eng. pdf). Accessed 10 December 2015.

15. Seddon JA, Shingadia D. Epidemiology and disease burden of tuberculosis in children: a global perspective. Infection and Drug Resistance 2014; 7: 153-165.

16. Donald PR, Marais BJ, Barry 3rd CE. Age and the epidemiology and pathogenesis of tuberculosis. Lancet 2010; 375: 1852-1854.

17. Dye C. The Population Biology of Tuberculosis. Princeton: Princeton University Press, 2015, pp. 18-25 (Levin SA, Hom HS, eds. Monographs in Population Biology).
18. Horna-Campos OJ, et al. Public transportation and pulmonary tuberculosis, Lima, Peru. Emerging Infectious Diseases 2007; 13: 1491-1493.

19. Borgdorff MW, et al. Transmission of tuberculosis between people of different ages in The Netherlands: an analysis using DNA fingerprinting. International Journal of Tuberculosis and Lung Disease 1999; 3: 202-206.

20. World Health Organization. Treatment of tuberculosis guidelines, 4th edn. Geneva: World Health Organization (http://apps.who.int/iris/bitstream/10665/ 44165/1/9789241547833_eng.pdf). Accessed 10 December 2015.

21. Omori S. Current status and problems of tuberculosis control in Japan (11). Survey to find the patterns of tuberculosis incidences - the past and future [in Japanese]. Nihon Koshu Eisei Zasshi 2009; 56: 530-534.

22. World Health Organization. Global TB database (http:// www.who.int/tb/country/data/download/en/). Accessed 10 September 2015.

23. Saito K, et al. (eds). A Recent Advance in Time Series Analysis by Maximum Entropy Method-Applications to Medical and Biological Sciences. Sapporo: Hokkaido University Press, 1994.

24. Ohtomo $\mathbf{N}$, et al. New method of time series analysis and its application to Wolf's sunspot number data. Japanese Journal of Applied Physics 1994; 33: 23212831.

25. Omori M, et al. Current epidemiological trend of tuberculosis in Japan. International Journal of Tuberculosis and Lung Disease 2002; 6: 415-423.

26. Aoshima S. Perfect Guide to Treatment of Tuberculosis [in Japanese]. Tokyo: Chyu-gai-igakusha, 2015, pp. 189.

27. Japan Ministry of Health and Welfare. Statistics of TB 1999-2014 [in Japanese].

28. The Tuberculosis Surveillance Center. (http://www.jata. or.jp/rit/ekigaku/). Accessed 10 September 2015.

29. Sumi A, et al. Time-series analysis of hepatitis A, B, C and $\mathrm{E}$ infections in a large Chinese city: application to prediction analysis. Epidemiology and Infection 2013; 141: 2666-2678.

30. Sumi A, et al. Effect of temperature, relative humidity and rainfall on rotavirus infections in Kolkata, India. Epidemiology and Infection 2013; 141: 1652-1661.

31. Armitage P, Berry G, Matthews JNS. Statistical Methods in Medical Research, 4th edn. Oxford: Blackwell Science, 2012.

32. Sumi A, et al. Prediction analysis for measles epidemics. Japan Journal of Applied Physics 2003; 42: 7611-7620.

33. Harigane $\mathbf{K}$, et al. The role of temperature in reported chickenpox cases from 2000 to 2011 in Japan. Epidemiology and Infection 2014; 143: 2666-2678.

34. Hoshino H, Uchimura K, Yamauchi Y. Comparison of $\mathrm{TB}$ incidence of young and middle age groups between urban/suburban prefectures and other prefectures [in Japanese]. Kekkaku 2009; 84: 1-8.

35. Bratescu A, Teodorescu M. Circannual variations in the $\mathrm{B}$ cell/T cell ratio in normal human peripheral 
blood. Journal of Allergy Clinical Immunology 1981; 68: 273-280.

36. Yamamoto S, et al. Seasonal variationin the incidence of pulmonary tuberculosis among the elderly in the Kanto area and its meteorological factors [in Japanese]. Japan Journal of Biometeorology 2003; 40: 83-92.

37. ten Asbroek AHA, et al. Estimation of serial interval and incubation period of tuberculosis using DNA fingerprinting. International Journal of Tuberculosis and Lung Disease 1999; 3: 414-420.

38. Toyota $\mathbf{E}$, et al. Clinical investigation among elderly patients with tuberculosis [in Japanese]. Kekkaku 2010; 85: $655-660$.

39. Keiichi T. Tuberculosis and the Japanese. Tokyo: Iwanami Shoten, 2011.

40. Rahman M, et al. BCG vaccination and tuberculosis in Japan. Journal of Epidemiology 2003; 13: 127-135. 